INPLASY

PROTOCOL

To cite: Guo et al. Evaluation of the effectiveness and safety of cupping therapy in the treatment of asthma : A protocol for systematic review and meta-analysis. Inplasy protocol 202180104. doi: 10.37766/inplasy2021.8.0104

Received: 26 August 2021

Published: 26 August 2021

Corresponding author: Lie Wang

slpwzt7063@163.com

Author Affiliation: Changchun University of Chinese Medicine.

Support: No.

Review Stage at time of this submission: The review has not yet started.

Conflicts of interest: None declared.

\section{Evaluation of the effectiveness and safety of cupping therapy in the treatment of asthma : A protocol for systematic review and meta-analysis}

Guo, L1; Wang, ZT2; Wei, LN3; Ding, LZ4; Kong, YB5; Liu, ZM6; Tian, Y7; Yang, FS8; Wang, L9; Sun, LP10.

Review question / Objective: "The aim of this systematic review is to compare Cupping therapy and Non cupping therapy in terms of efficacy and acceptability in the asthma to better inform clinical practice. To this end, the proposed systematic review will address the following question: Which is the best choice to reduce PEF, FVC, and FEV1, in asthma patients, Cupping therapy or Non cupping therapy ?". Information sources: The protocol was prepared based on the preferred reporting project of the systematic review and meta-analysis protocol statement guidelines. Our research does not require ethical approval, as all analyses will be based on aggregated data from previously published studies. We will search the following Chinese and English databases: China National Knowledge Infrastructure, China Science and Periodical Database, Wanfang Database, China Biomedical Literature Database, PubMed, Embase, Cochrane Library.All of the above electronic databases will be searched from inception to August 22, 2021. In addition, we will manually search for conference papers, ongoing experiments and internal reports to supplement the studies retrieved via electronic search.

INPLASY registration number: This protocol was registered with the International Platform of Registered Systematic Review and Meta-Analysis Protocols (INPLASY) on 26 August 2021 and was last updated on 26 August 2021 (registration number INPLASY202180104).

\section{INTRODUCTION}

Review question / Objective: "The aim of this systematic review is to compare Cupping therapy and Non cupping therapy in terms of efficacy and acceptability in the asthma to better inform clinical practice. To this end, the proposed systematic review will address the following question: Which is the best choice to reduce PEF, FVC, and 
FEV1, in asthma patients, Cupping therapy or Non cupping therapy ?".

Condition being studied: Asthma is one of the most common chronic airway diseases and is characterized by wheezing, dyspnea, chest tightness and coughing [1]. These symptoms reduce the patientl's quality of life and limit physical activity in daily life [2]. At present, approximately 334 million people worldwide suffer from asthma, and its incidence is increasing rapidly at a rate of $20 \%-25 \%$ every ten years. It is estimated that the number of asthma patients will increase to 400 million in 2025, which will lead to a huge health and economic burden in the country [3]. Corticosteroids are conventional drugs for the treatment of bronchial asthma. However, despite the use of medium to high doses of inhaled corticosteroids and long-acting $\beta 2$ receptor agonists, many patients with severe asthma continue to experience asthma symptoms and worsening, leading to the emergence of refractory asthma [4]. In addition, the long-term use of steroid drugs will bring about a series of adverse reactions, such as local Candida infection, cataracts, glaucoma, osteoporosis, tachycardia, and hyperglycemia [5]. Existing studies have shown that cupping therapy can quickly improve the symptoms of asthma patients who are not sensitive to corticosteroids and bronchodilators and can be used as an alternative therapy [6]. However, there is no systematic review of the efficacy of cupping therapy in the treatment of asthma. To evaluate the efficacy and safety of cupping in the treatment of asthma, we conducted a systematic review and meta-analysis of published randomized clinical trials (RCTs) of cupping in the treatment of asthma.

\section{METHODS}

Search strategy: We will use \"cuppingl" and I"asthmal" as keywords to retrieve RCTs examining the treatment of asthma in Chinese and English databases.

Participant or population: "Asthma patients will be eligible for this review, with no exclusions based on ethnicity, gender or age."

Intervention: cupping therapy in the treatment group alone or in combination with other therapies.

Comparator: Does not include cupping therapy.

Study designs to be included: Randomized controlled trial.

Eligibility criteria: The inclusion criteria will be as follows: meeting the clinical diagnostic criteria for asthma, regardless of race, age and gender; since the establishment of the database, all Chinese and English versions of cupping therapy have been randomized controlled trials in the treatment of asthma; cupping therapy in the treatment group alone or in combination with other therapies, and the control group does not include cupping therapy; research articles that evaluate the maximum peak expiratory flow rate (PEF), forced vital capacity (FVC), forced expiratory volume in the first second (FEV1), asthma control questionnaire (ACQ) score, and asthma quality of life questionnaire (AQLQ) score. The exclusion criteria will be as follows: studies that do not meet the above inclusion criteria; incomplete or incorrectly researched data; patients with asthma who have other diseases or serious complications; case reports, comments or letters, biochemical tests, protocols, meeting abstracts and reviews.

Information sources: The protocol was prepared based on the preferred reporting project of the systematic review and metaanalysis protocol statement guidelines. Our research does not require ethical approval, as all analyses will be based on aggregated data from previously published studies. We will search the following Chinese and English databases: China National Knowledge Infrastructure, China Science and Periodical Database, Wanfang Database, China Biomedical Literature Database, PubMed, Embase, Cochrane Library.All of the above electronic 
databases will be searched from inception to August 22, 2021. In addition, we will manually search for conference papers, ongoing experiments and internal reports to supplement the studies retrieved via electronic search.

Main outcome(s): The main outcome indicators will be PEF, FVC, and FEV1, and the secondary outcome indicators will include ACQ score, AQLQ score and adverse events.

Quality assessment / Risk of bias analysis: Two researchers (YH and JW) will use the RCT bias risk assessment tool recommended by the Cochrane Collaboration Network bias risk assessment tool to assess the risk of bias among the included studies. Disagreements will be resolved by consulting a third researcher.

Strategy of data synthesis: We will use RevMan 5.4 software for the meta-analysis. We will use relative risk as an effective indicator of counting data and use the mean differences as an effective indicator of measurement data. The confidence interval for each effect index will be set to $95 \%$. Additionally, 12 will be used to quantitatively assess heterogeneity. If there is no statistical heterogeneity between the studies, a fixed effects model will be used for the meta-analysis. If there is heterogeneity, a random effects model will be used. $P<0.05$ indicates statistical significance.

Subgroup analysis: When there is potential heterogeneity in this study, if all the information included in the study was available, we could perform subgroup analysis based on the sex, age and treatment time of the included subjects.

Sensitivity analysis: A sensitivity analysis will be performed to assess the robustness of the included results. If the results are unstable, studies with a high risk of bias will be excluded.

Country(ies) involved: china.
Other relevant information: References 1.Global Initiative for Asthma (GINA). Global Strategy for Asthma Managem -ent and Prevention. 2021. http://www.ginasthma. org/reports. Accessed 5 May 2021. 2.Haselkorn T, Chen H, Miller DP, Fish JE, Peters SP, Weiss ST, et al. Asthma control and activity limitations: insights from the Real-world Evaluation of Asthma Control and Treatment (REACT) study. Ann Allergy Asthma Immunol 2010;104:471-7. 3.Reddel HK, Bateman BD, Becker A, et al. Asummary of the new GINA straegy: a roadmap to asthma control[J]. European Respiratory Journal, 2015;46(3): 622-639. 4.Chen S, Golam S, Myers J, Bly C, Smolen $H, X u X$. Systematic literature review of the clinical, humanistic, and economic burden associated with asthma uncontrolled by GINA steps 4 or 5 treatment. Curr Med Res Opin. 2018;34:2075-88. 5.Rodrigo G J, Castro-Rodriguez $\mathrm{J}$ A. Anticholinerg ics in the treatment of children and adults with acute asthma: a systematic review with meta-analysis. Thorax. 2005;60(9):740-746. 6.Goodwin Jodi,Mclvor R Andrew, Alternative therapy: cupping for asthma.Chest, 2011;139: 475-476.

Keywords: cupping; asthma; protocol; systematic review; meta-analysis.

Contributions of each author:

Author 1 - Lei Guo - The author participates in conception evaluation, design evaluation, coordination evaluation, data collection, data management, data analysis, interpretation of data and preparation of scheme.

Email: 1187623487@qq.com

Author 2 - Zhongtian Wang - The author participates in conception evaluation, design evaluation, data management and scheme writing.

Author 3 - Lina Wei - The author participates in conception evaluation, coordination evaluation, data collection, data analysis, data interpretation and scheme preparation.

Author 4 - Lizhong Ding - The authors are involved in conceptualizing evaluations, coordinating evaluations, data collection, interpreting data and writing programs. 
Author 5 - Yibu Kong - The author participates in design evaluation, coordination evaluation, interpretation of data and preparation of schemes.

Author 6 - Zhimei Liu - The author participates in design evaluation, coordination evaluation, interpretation of data and preparation of schemes.

Author 7 - Ye Tian - The author is involved in data collection, data management, data analysis and interpretation.

Author 8 - Fushuang Yang - The author is involved in data collection, data management, data analysis and interpretation.

Author 9 - Lie Wang - The author participates in conception evaluation, design evaluation, preparation evaluation and financial support.

Email: slpwzt7063@163.com

Author 10 - Liping Sun - The author participates in conception evaluation, design evaluation, preparation evaluation and financial support. 